\title{
Bribing potential entrants in a rent-seeking contest
}

\author{
Lambert Schoonbeek
}

Received: 2 June 2008 / Accepted: 12 November 2008 / Published online: 18 November 2008

(C) The Author(s) 2008. This article is published with open access at Springerlink.com

\begin{abstract}
We consider a two-stage model of a Tullock rent-seeking contest where one new potential entrant makes his appearance. In the first stage each other player can contribute to bribe this new player to commit not to enter the contest. In the second stage we have the actual contest either with or without the new player. We present the conditions such that there exist equilibria in which the new player is being bribed.
\end{abstract}

Keywords Rent-seeking contest · Entry $\cdot$ Bribing

\section{JEL Classification D7}

\section{Introduction}

Tullock (1980) introduced the rent-seeking contest where a number of risk-neutral players compete for a single indivisible prize. Each player can increase his probability of winning the prize by exerting a nonrefundable effort. A well-known application of the model is that the players, say firms, lobby for a monopoly rent which is awarded by the contest organizer, say the government. The model has spawned a large literature, see Nitzan (1994), Lockard and Tullock (2001), Konrad (2007) and Congleton et al. (2008). Matros (2006) carefully examined the effects of the addition (or deletion) of one extra player on both the individual and aggregate efforts and the winning probabilities in equilibrium. He has not analyzed the effects on equilibrium payoffs. ${ }^{1}$

This paper also examines the effects of adding a new player to a rent-seeking contest. However, in contrast to Matros (2006), we suppose that the new player might be bribed to

\footnotetext{
${ }^{1}$ Higgins et al. (1985) investigated the equilibrium number of rent-seekers and aggregate rent-seeking efforts in a situation with free entry in both Tullock's (1980) contest and a more general contest. However, bribing does not play a role in their analysis.
}

L. Schoonbeek $(\bowtie)$

Department of Economics and Econometrics, University of Groningen, P.O. Box 800,

9700 AV Groningen, The Netherlands

e-mail: L.Schoonbeek@rug.nl 
commit not to enter the contest. We have a two-stage model. In stage 1, each incumbent player (who participates in the contest anyway) noncooperatively decides how much to contribute to bribe the new player. If the summation of all contributions is at least as large as the reservation payoff of the new player, this player will be bribed, retain all offers and exit. Otherwise, the contributions are refunded to the incumbents, and the new player will enter. In stage 2, we have the actual contest either with or without the new player, depending on what has happened in stage 1 . We present conditions such that the model has (pure-strategy subgame-perfect) equilibria in which the new player is being bribed.

Stage 1 of our model is related to private voluntary contribution games for public goods as analyzed by Bagnoli and Lipman (1989) and Cadsby and Maynes (1999). The reason is that each incumbent, even if he has contributed nothing to bribe the new player, will benefit if the latter stays away. This gives each incumbent an incentive to free ride on the contributions of others. Our work is further related to recent papers on corruption and collusion in auctions. See Esô and Schummer (2004), who study a second-price auction where one player can bribe the other player to commit not to participate in the auction (see further Chen and Tauman 2006). Note that we have a peculiar form of collusion, wherein the primary beneficiaries may not be one of the colluders, but the non-bribing players.

In Sect. 2 we outline the model and investigate its equilibria. We conclude in Sect. 3. Proofs are in the Appendix.

\section{The model and results}

Consider a standard Tullock rent-seeking contest for a prize in the following two-stage model. There are $n$ incumbent players $(n \geq 2)$ who will compete in the contest regardless. There is a (potential) entrant, player $n+1$, who contemplates participating in the contest. All players are risk neutral. The observable valuation of the prize by player $i=1, \ldots, n+1$ is $v_{i}>0$. Without loss of generality we assume that $v_{1} \geq v_{2} \geq \cdots \geq v_{n}>0$ and that the unit of money is such that $\sum_{j=1}^{n} \frac{1}{v_{j}}=1$. The latter implies that $v_{i}>1$ for $i=1, \ldots, n$. Notice that both $v_{n+1} \leq v_{n}$ and $v_{n+1}>v_{n}$ are possible. In stage 1 , each incumbent decides how much to contribute to bribe player $n+1$ to stay away. Suppose that incumbent $i$ contributes some fraction $z_{i} \geq 0$ of the reservation payoff of player $n+1$, where this payoff is defined as the equilibrium payoff player $n+1$ will obtain in case he enters the contest. If $\sum_{j=1}^{n} z_{j} \geq 1$, player $n+1$ is being bribed, i.e., he retains all contributions and exits (he may keep excess contributions). If $\sum_{j=1}^{n} z_{j}<1$, player $n+1$ will not be bribed and enters the contest, while the contributions are refunded to the incumbents without further costs. In stage 2 , we have the actual contest for the prize among the $n$ incumbents if player $n+1$ has been bribed. Otherwise, the contest is among all $n+1$ players. We assume that in stage 1 the organizer of the contest observes neither the players nor the possible bribing of the entrant; however he observes all active players in stage 2 . We find equilibria of this model applying backward induction.

Consider stage 2 if the entrant has been bribed. The probability that player $i=1, \ldots, n$ wins the prize is $p_{i, n}=x_{i, n} / \sum_{j=1}^{n} x_{j, n}$, with $x_{j, n} \geq 0$ the nonrefundable effort of player $j$. The second subindex ' $n$ ' attached to a variable indicates that we consider the contest with $n$ players. If all players expend zero effort, each player wins the prize with probability $1 / n$. Player $i$ maximizes his (expected) payoff

$$
\pi_{i, n}=\frac{x_{i, n} v_{i}}{\sum_{j=1}^{n} x_{j, n}}-x_{i, n}
$$


Using Stein (2002), we know that in the (unique) equilibrium player $i$ exerts effort $\hat{x}_{i, n}=$ $s_{n}\left(1-\frac{s_{n}}{v_{i}}\right)$, where $s_{n} \equiv(n-1) / \sum_{j=1}^{n} \frac{1}{v_{j}}=n-1$ (throughout we denote equilibrium values with a hat). Hence, his equilibrium payoff is $\hat{\pi}_{i, n}=\frac{1}{v_{i}}\left(v_{i}-s_{n}\right)^{2}=\frac{1}{v_{i}}\left(v_{i}-n+1\right)^{2}$. So far, we have assumed that all $n$ players are active in equilibrium, i.e., exert a positive effort. Using Stein (2002), this is true if and only if the smallest valuation is 'large enough', i.e., $v_{n}>(n-2) / \sum_{j=1}^{n-1} \frac{1}{v_{j}}$, or equivalently $v_{n}>n-1$.

Next, consider stage 2 if the entrant has not been bribed. Player $i=1, \ldots, n+1$ maximizes $\pi_{i, n+1}$, where the subindex ' $n+1$ ' means that we have the contest with $n+1$ players. The equilibrium effort and payoff of player $i$ are $\hat{x}_{i, n+1}=s_{n+1}\left(1-\frac{s_{n+1}}{v_{i}}\right)$ and $\hat{\pi}_{i, n+1}=\frac{1}{v_{i}}\left(v_{i}-s_{n+1}\right)^{2}$, where $s_{n+1} \equiv n / \sum_{j=1}^{n+1} \frac{1}{v_{j}}$. If $v_{n+1} \leq v_{n}$, all players are active in equilibrium if and only if $v_{n+1}>(n-1) / \sum_{j=1}^{n} \frac{1}{v_{j}}=n-1$. If $v_{n+1}>v_{n}$, all players are active in equilibrium if and only if $v_{n}>(n-1) /\left(\sum_{j=1}^{n-1} \frac{1}{v_{j}}+\frac{1}{v_{n+1}}\right)$.

We present Assumption 1 and Lemma 1.

Assumption 1 All players are active in the equilibrium in stage 2 of both the contest with $n$ players and $n+1$ players.

Lemma 1 Assumption 1 is equivalent to stating that one of the following three situations holds: (1) $n-1<v_{n+1} \leq v_{n}$; (2) $v_{1}=v_{n}$ and $v_{n}<v_{n+1}$; (3) $v_{1}>v_{n}$ and $n-1<v_{n}<$ $v_{n+1}<\frac{v_{n}}{n-v_{n}}$.

We will impose Assumption 1 from now on. Notice that if player $n+1$ would not be active in the contest with $n+1$ players, his appearance would have no consequences, which is not interesting. ${ }^{2}$

Proceeding, examine $\Delta_{i} \equiv \hat{\pi}_{i, n}-\hat{\pi}_{i, n+1}$ for $i=1, \ldots, n$, i.e., the difference for incumbent $i$ between the payoff in the equilibrium of stage 2 with respectively $n$ and $n+1$ players. We have $\Delta_{i}=\frac{1}{v_{i}}\left(v_{i}-s_{n}\right)^{2}-\frac{1}{v_{i}}\left(v_{i}-s_{n+1}\right)^{2}=\frac{s_{n}^{2}-s_{n+1}^{2}}{v_{i}}+2\left(s_{n+1}-s_{n}\right)$. We know from Matros (2006) that $v_{i}>s_{n+1}>s_{n}>0$ for each $i$. Hence, $\Delta_{1} \geq \Delta_{2} \geq \cdots \geq \Delta_{n}>0$. Notice that entry of player $n+1$ hurts all incumbents.

Next, we turn to stage 1 where each incumbent decides how much to contribute to bribe player $n+1$. Recall that player $n+1$ will be bribed if $\sum_{j=1}^{n} z_{j} \geq 1$. Hence, incumbent $i$ selects $z_{i}$ while facing payoff function $\hat{\pi}_{i, n}-z_{i} \hat{\pi}_{n+1, n+1}$ if $\sum_{j=1}^{n} z_{j} \geq 1$, and $\hat{\pi}_{i, n+1}$ if $\sum_{j=1}^{n} z_{j}<1$. Define $z_{-i}=\sum_{j \neq i} z_{j}$. Obviously, incumbent $i$ will never select $z_{i}>1$. If $z_{-i} \geq 1$, the best response of incumbent $i$ equals $z_{i}=0$. If $0 \leq z_{-i}<1$, the contribution $z_{i}=1-z_{-i}$ is (just) enough to bribe player $n+1$. This is optimal for incumbent $i$ if and only if $\Delta_{i} \geq\left(1-z_{-i}\right) \hat{\pi}_{n+1, n+1}$. Proposition 1 characterizes the best response of incumbent $i$ if $0 \leq z_{-i}<1$.

Proposition 1 Let Assumption 1 hold. Consider incumbent $i=1, \ldots, n$. Let $0 \leq z_{-i}<1$ be given. In stage $1, z_{i}=1-z_{-i}$ is the best response of incumbent $i$ if both (i) $v_{n+1}<f\left(1-z_{-i}\right)$

\footnotetext{
${ }^{2}$ One might also consider the case where in the $n$-player contest some incumbents remain inactive in equilibrium. However, such incumbents will never bribe the new player, which makes this case less interesting. Next, it might also happen that in the $(n+1)$-player contest, some incumbents become inactive in equilibrium. Analysis of bribing along the lines of Proposition 1 becomes analytically cumbersome in this case and does not yield new insights.
} 
and (ii) $v_{i} \geq g\left(1-z_{-i}, v_{n+1}\right)$ hold. Else any $z_{i} \in\left[0,1-z_{-i}\right)$ is a best response. Here, with $0<z \leq 1$, we have:

$$
\begin{aligned}
f(z) & =\frac{(n-1) z+2+\sqrt{((n-1) z+2)^{2}+8 z}}{2 z}, \\
g\left(z, v_{n+1}\right) & =\frac{(2 n-1) v_{n+1}+n-1}{((n-1) z+2) v_{n+1}+2-z v_{n+1}^{2}} .
\end{aligned}
$$

Thus, given $0 \leq z_{-i}<1$, incumbent $i$ makes a contribution which is sufficient to bribe player $n+1$ if and only if $v_{n+1}$ is 'small enough', and moreover $v_{i}$ is 'large enough'. The threshold for $v_{n+1}$ depends only on $z_{-i}$ and $n$; the threshold for $v_{i}$ depends on $z_{-i}, v_{n+1}$ and $n$. It can be verified that $f(z)$ is decreasing in $z$, while $g\left(z, v_{n+1}\right)$ is increasing in both $z$ and $v_{n+1}$. These effects correspond to intuition. If $z_{-i}$ increases, conditions (i) and (ii) of Proposition 1 are relaxed. If $v_{n+1}$ increases, condition (ii) becomes tighter.

Observe that equilibria with bribing are characterized by the following two conditions: (a) $\sum_{j=1}^{n} \hat{z}_{j}=1$, and (b) $\Delta_{i} \geq \hat{z}_{i} \hat{\pi}_{n+1, n+1}$ for $i=1, \ldots, n$. Clearly, if $\sum_{j=1}^{n} \Delta_{j}>\hat{\pi}_{n+1, n+1}$, there exist many of these equilibria. However, besides these, there may also exist multiple equilibria without bribing. Suppose, e.g., that $\Delta_{1}<\hat{\pi}_{n+1, n+1}$. We then have such equilibria if $\sum_{j=1}^{n} \hat{z}_{j}<1$ and $\sum_{j \neq i} \hat{z}_{j} \hat{\pi}_{n+1, n+1}+\Delta_{i}<\hat{\pi}_{n+1, n+1}$ for $i=1, \ldots, n$. Notice that these (inefficient) equilibria without bribing are caused by free-riding behavior of the incumbents, cf. Bagnoli and Lipman (1989).

Using Proposition 1 we can easily find necessary and sufficient conditions for the existence of specific equilibria with bribing. We focus on three interesting examples. First, we have an equilibrium with bribing in which each incumbent makes a contribution proportional to his own valuation (i.e., $\hat{z}_{i}=\frac{v_{i}}{\sum_{j} v_{j}}$ for all $i$ ) if and only if $v_{n+1}<f\left(\frac{v_{1}}{\sum_{j} v_{j}}\right)$ and $v_{i} \geq g\left(\frac{v_{i}}{\sum_{j} v_{j}}, v_{n+1}\right)$ for $i=1, \ldots, n .^{3}$ Second, there is an equilibrium with bribing where the contribution is identical for each incumbent (i.e., $\hat{z}_{i}=\frac{1}{n}$ for all $i$ ) if and only if $v_{n+1}<f\left(\frac{1}{n}\right)$ and $v_{n} \geq g\left(\frac{1}{n}, v_{n+1}\right)$. If $v_{1}=\cdots=v_{n}=v_{n+1}$, such an equilibrium exists for all $n \geq 2 .{ }^{4}$ Third, there exists an equilibrium where a single incumbent $i$ bribes player $n+1$ (i.e., $\hat{z}_{i}=1$ and $\hat{z}_{j}=0$ for $j \neq i$ ) if and only if $v_{n+1}<f(1)$ and $v_{i} \geq g\left(1, v_{n+1}\right)$. Interestingly, if $v_{1}=\cdots=v_{n}=v_{n+1}$, such an equilibrium only exists for $n=2$.

Matros (2006) has shown that the aggregate equilibrium efforts always strictly increase if an additional active player enters the contest. Thus, bribing hurts the organizer of the contest if he is interested in maximizing aggregate efforts. Recall however that in our model the organizer cannot observe possible bribing in stage 1 .

\section{Conclusion}

We have analyzed a two-stage rent-seeking contest where a new player might be bribed to stay away. We have presented conditions such that bribing occurs in equilibrium. However, due to free-riding behavior, we may also have equilibria without bribing. For simplicity, we assumed that the incumbents have all the bargaining power, i.e., the new player accepts the

\footnotetext{
${ }^{3}$ Notice that we have $f\left(\frac{v_{1}}{\sum_{j} v_{j}}\right) \leq f\left(\frac{v_{2}}{\sum_{j} v_{j}}\right) \leq \cdots \leq f\left(\frac{v_{n}}{\sum_{j} v_{j}}\right)$.

${ }^{4}$ Notice using $\sum_{j=1}^{n} \frac{1}{v_{j}}=1$, that $v_{i}=n$ for $i=1, \ldots, n+1$ in this case.
} 
bribing contributions in case he receives at least his (minimum) reservation payoff. However, qualitatively the results do not depend on this assumption. Finally, note that bribery will be hard to observe for a researcher, since players will hide such behavior. Yet, our results show that it can prevail in equilibrium.

Acknowledgement I thank an anonymous referee, Allard van der Made and Bastiaan Overvest for very helpful suggestions.

Open Access This article is distributed under the terms of the Creative Commons Attribution Noncommercial License which permits any noncommercial use, distribution, and reproduction in any medium, provided the original author(s) and source are credited.

\section{Appendix: Proofs}

Proof of Lemma 1 We distinguish two cases: (i) $v_{n+1} \leq v_{n}$, and (ii) $v_{n+1}>v_{n}$. In case (i) we directly obtain that all relevant players are active in both contests if and only if $v_{n+1}>n-1$. Considering case (ii), we distinguish two subcases: (iia) $v_{1}=v_{n}$, and (iib) $v_{1}>v_{n}$. Using $\sum_{j=1}^{n} \frac{1}{v_{j}}=1$, we have $v_{n}=n$ in subcase (iia), and thus all relevant players are always active in both contests. In subcase (iib) we have $v_{n}<n$. Using this, we obtain that all players are active in the contest with $n+1$ players if and only if $v_{n}>(n-1) /\left(1-\frac{1}{v_{n}}+\frac{1}{v_{n+1}}\right)$ or $v_{n+1}<\frac{v_{n}}{n-v_{n}}$. The lemma follows directly.

Proof of Proposition 1 Consider the inequality $\Delta_{i} \geq z \hat{\pi}_{n+1, n+1}$ for $0<z \leq 1$. Using $\sum_{j=1}^{n+1} \frac{1}{v_{j}}=\left(v_{n+1}+1\right) / v_{n+1}$, it can be rewritten as

$$
\frac{1}{v_{i}}\left(n-1+\frac{n v_{n+1}}{v_{n+1}+1}-2 v_{i}\right)\left(n-1-\frac{n v_{n+1}}{v_{n+1}+1}\right) \geq \frac{z}{v_{n+1}}\left(v_{n+1}-\frac{n v_{n+1}}{v_{n+1}+1}\right)^{2}
$$

or

$$
\left(2 v_{i}-n+1+\left(2 v_{i}+1-2 n\right) v_{n+1}\right)\left(v_{n+1}-n+1\right) \geq\left(v_{n+1}-n+1\right)^{2} z v_{i} v_{n+1} .
$$

Using Assumption 1, we always have $v_{n+1}-n+1>0$. As a result, (A.1) can be simplified to $v_{i}\left[(2+n z-z) v_{n+1}+2-z v_{n+1}^{2}\right] \geq(2 n-1) v_{n+1}+n-1$. Notice that $v_{i}>0$ and $(2 n-1) v_{n+1}+n-1>0$ since $n \geq 2$. Hence, we must have $h\left(v_{n+1}\right) \equiv(2+n z-z) v_{n+1}+$ $2-z v_{n+1}^{2}>0$ as well. Note that $h(n-1)=2 n>0$. The unique positive root of $h\left(v_{n+1}\right)=0$ equals $v_{n+1}=\frac{(n-1) z+2+\sqrt{((n-1) z+2)^{2}+8 z}}{2 z}(>n-1)$. Thus, $h\left(v_{n+1}\right)>0$ for $v_{n+1}>0$ if and only if $v_{n+1} \in\left(n-1, \frac{(n-1) z+2+\sqrt{((n-1) z+2)^{2}+8 z}}{2 z}\right)$. The proposition follows easily.

\section{References}

Bagnoli, M., \& Lipman, B. L. (1989). Provision of public goods: fully implementing the core through private contributions. Review of Economic Studies, 56, 583-601.

Cadsby, C. B., \& Maynes, E. (1999). Voluntary provision of threshold public goods with continuous contributions: experimental evidence. Journal of Public Economics, 71, 53-73.

Chen, C., \& Tauman, Y. (2006). Collusion in one-shot second-price auctions. Economic Theory, 28, 145-172.

Congleton, R. D., Hillman, A. L., \& Konrad, K. A. (Eds.) (2008). 40 Years of research on rent seeking, volumes I and II. Berlin: Springer. 
Eső, P., \& Schummer, J. (2004). Bribing and signaling in second price auctions. Games and Economic Behavior, 47, 299-324.

Higgins, R. S., Shughart II, W. F., \& Tollison, R. D. (1985). Free entry and efficient rent seeking. Public Choice, 46, 247-258.

Konrad, K. A. (2007). Strategy in contests - an introduction (Discussion Paper SP II 2007-01). Wissenschaftszentrum Berlin.

Lockard, A. L., \& Tullock, G. (Eds.) (2001). Efficient rent-seeking: chronicle of an intellectual quagmire. Dordrecht: Kluwer Academic.

Matros, A. (2006). Rent-seeking with asymmetric valuations: addition or deletion of a player. Public Choice, $129,369-380$.

Nitzan, S. (1994). Modelling rent-seeking contests. European Journal of Political Economy, 10, 41-60.

Stein, W. E. (2002). Asymmetric rent-seeking with more than two contestants. Public Choice, 113, 325-336.

Tullock, G. (1980). Efficient rent seeking. In J. M. Buchanan, R. D. Tollison, \& G. Tullock (Eds.), Toward a theory of the rent-seeking society (pp. 97-112). College Station: Texas A\&M University Press. 